\title{
A Robot Emotion Generation Mechanism Based on PAD Emotion Space
}

\section{Research on Robot Emotion}

\author{
Gao Qingji*, Wang Kai* and Liu Haijuan** \\ * Robotics Institute,Civil, Aviation University of China \\ Tianjin, 300300, China \\ wangkaiblue@163.com \\ ** Department of Automation, Northeast Dianli University \\ Jilin, 132012, China \\ cherygir10829@yahoo.com.cn
}

\begin{abstract}
A robot emotion generation mechanism is presented in this paper, in which emotion is described in PAD emotion space. In this mechanism, emotion is affected by the robot personality, the robot task and the emotion origin, so the robot emotion will change naturally when it senses the extern stimuli. We also experiment on Fuwa robot, and demonstrate that this mechanism can make the robot's emotion change be more easily accepted by people and is good for humanrobot interaction.
\end{abstract}

KEYWORDS: artificial emotion, emotion space, OCC emotion model

\section{Introduction}

As the development of cognitive science, neuroscience and psychology, the research results show that emotion plays a crucial role in attention, planning, reasoning and decision-making. It was probably in 1981, that, for the first time, Sloman[7] proposed an idea that "the need to cope with a changing and partly unpredictable world makes it very likely that any intelligent system with multiple motives and limited powers will have emotions". In the society of mind, Minsky [3], noted that "The question is not whether intelligent machines can have any emotions, but whether machines can be intelligent without any emotions".

Robot endowed with emotion can enhance the performance of its behaviour. In recent years, researchers of robotics are being actively conducted to develop robotics that can help a user to do a desired job so as to accommodate the convenience of the user. Special interests are being taken to develop an intelligent robot that can make an intelligent determination through an interaction with a user 
and can perform a corresponding operation[4]. If robots make decision without emotions, they decide solely using mechanisms based on reason. Examples include planning algorithms and state machines etc.[4], and robot in those mechanisms cannot show its "feeling". As Picard argued[5], emotions influence the decision-making, learning and other cognitive functions of human. So, it is very significant if we equip robots with emotion, and the human-robot interaction (HRI) will be more natural.

Presently, researchers in artificial intelligence have some previous work in emotion robot; however, most of them are still in the phase of research, we cannot directly apply to the real system. The research of artificial emotion can be generalized to three aspects: emotion recognition, emotion expression and the emotion control architecture [6,8,9]; the emotion control architecture is the essential part of artificial emotion, and the emotion generation mechanism is the key of the emotion control architecture. Emotion generation mechanism mainly involves that how the robot's emotion changes when it responds to the environmental stimuli and how the variation of emotion can be easily accepted by human.

There ere several emotion generation mechanisms in this field. According to Picard[5], the affective computing model (it is assumed that the affect equals to the emotion, although there is some difference between them) can be divided into three types: discrete state model, emotion space model and the model based rules. However, the first two types are usually used in the practical system. The OCC model is a typical discrete state model (this model addressed that 22 emotion words related to real interaction), which computes the current emotion based on the event, object, and the agent state. The advantage of the model is that it considered the origin of emotions; The disadvantage of the model is that it can only describe the 22 kinds of emotion and it has the limitation of representing the strength or the intensity of emotion[12]. The computing model of emotion space commonly describes emotion in several abstract dimensions, so emotion can be easily represented in mathematics. The demerit of this model is that origin of emotion is not considered[12]. Wei Zhehua[11] presents a 3D emotion space generated by fear, indignation and relish, and any emotion can be represented in the combination of the 3D above. Broekens J. and D. DeGroot[1] employ the Pleasure-Arousal-Dominance (PAD) three dimensions to quantify emotion.

In order to make robot has an emotion generation method similar to that of human and enhance the flexible behaviour of robot in HRI, a robot emotion generation mechanism is proposed, and the merits of OCC model and PAD emotion space are adopted in this mechanism. The proposed mechanism is also applied in the practical system (Fuwa robot designed for the Beijing $200829^{\text {th }}$ Olympic Games, as Fig 1.1) to show the effect of emotion. This paper is organized as follows: section 2 introduces the representation of emotion and personality; Section 3 shows the architecture of emotion generation mechanism; Section 4 shows the experiment with the proposed mechanism, and conclusions are given in Section 5. 
Fig 1.1 robot Jingjing

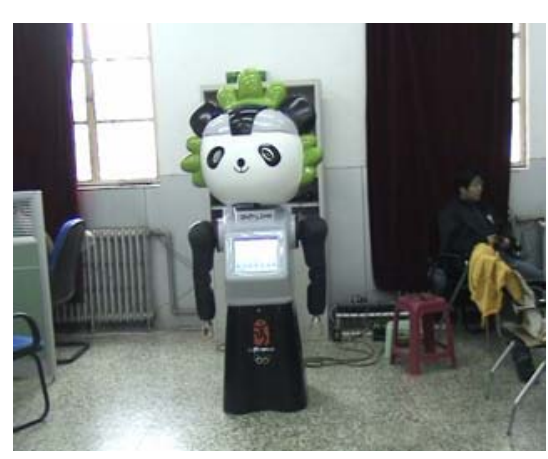

\section{Related Theory of Artificial Emotion}

\subsection{Representations of Emotion}

It is very critical to define some kind of measures to describe each emotion state in emotion generation mechanism. This paper chooses PAD emotion space designed by Albert Mehrabian (1974) [1]. The PAD scale determines emotions using a three-dimensional emotion space with the axes representing pleasure, arousal, and dominance (with possible values between -1 and 1 ). Pleasure represents the overall joy of the agent, and arousal represents its desire to interact with the world, and dominance represents its feeling of control in the situation.

This model is selected for two reasons:

1) The PAD three dimensions are nearly orthogonal scales of emotion, and Mehrabian argues that any emotion can be expressed in terms of values on these three dimensions, and provides extensive evidence for this claim.

2) Any two similar types of emotion in PAD emotion space can be differentiated easily, while it is hard in other emotion space.

Mehrabian provides an extensive list of emotional labels for points in the PAD space (Fig 2.1), which gives an impression of the emotional meaning of combinations of pleasure, arousal and dominance. 


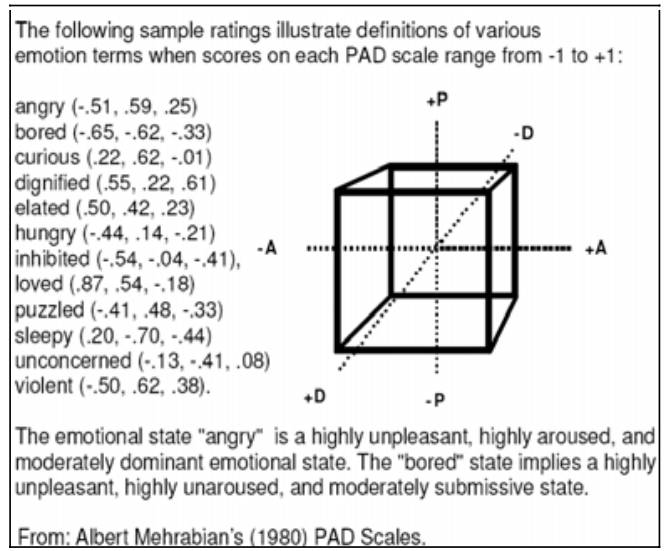

Fig 2.1 the Mehrabian P-A-D emotion space

In the PAD space, emotion state at time $t$ can be represented as emotion vector:

$\mathbf{E}_{t}=\left(\mathrm{P}_{t}, \mathrm{~A}_{\mathrm{t}}, \mathrm{D}_{t}\right)$.

\subsection{Description of Personality}

The human personality is affected by genetic factor and environmental factor. As a species our brains are almost identical which gives rise to our common sets of behaviours; However, because we are all genetically and environmentally unique, we are all different to varying degrees. It is these differences that give us our unique behavioural variations to common behaviour patterns. This paper assumes that the role of personality is to adjust the effect of the extern stimuli to robot emotion.

There are several methods to describe personality[10]. In fact, The OCEAN (Openness, Conscientiousness, Extraversion, Agreeableness, Neuroticism) 5D model and the EFA (Extroversion, Fear, Aggression) 3D model are often used. Each dimension of the two models describes the special nature of personality.

We select the extraversion, neuroticism, and fear three dimensions to describe personality. Each dimension value varies between -1 and 1 , and the extent of transfer correlated to the value of the dimension. The robot personality can be represented as follows:

$$
\mathbf{P}=(E, N, F)
$$

Let $\Delta \mathbf{E}_{\mathrm{t}}$ is objective change of robot emotion at time $t$ caused by extern event, and after weighted by robot's personality, objective emotion change $\Delta \mathbf{E}_{t}$ is converted into robot's emotion change $\Delta \mathbf{E}_{t}^{\prime}$. Equations $(2.1 \sim 2.3)$ describe the detail of personality effect.

$$
\begin{aligned}
& \Delta \mathbf{E}_{\mathrm{t}}^{\prime}=\mathrm{P}_{\mathrm{c}}\left(\Delta \mathbf{E}_{\mathrm{t}}\right) \\
& \Delta \mathbf{E}_{t}=\left(\Delta P_{t}, \Delta A_{t}, \Delta D_{t}\right) \\
& \Delta \mathbf{E}_{t}^{\prime}=\left(\Delta P_{t}^{\prime}, \Delta A_{t}^{\prime}, \Delta D_{t}^{\prime}\right)
\end{aligned}
$$

Where $\mathrm{P}_{c}$ stands for the personality transformation, formula (2.4) implements this transformation. 
$\mathrm{P}_{\mathrm{c}}\left(\Delta \mathbf{E}_{\mathrm{t}}\right)=\left[\begin{array}{ccc}1+1 / \mathrm{N} & 0 & 0 \\ 0 & 1+1 / \mathrm{N} & 0 \\ 0 & 0 & 1 / \mathrm{F}-1\end{array}\right]\left(\begin{array}{c}\mathrm{E} \\ \mathrm{N} \\ \mathrm{F}\end{array}\right)\left[\begin{array}{ccc}1 & 0 & 0 \\ 0 & 1 & 0 \\ 0 & 0 & 1\end{array}\right]\left(\begin{array}{c}\Delta \mathrm{P}_{\mathrm{t}} \\ \Delta \mathrm{A}_{\mathrm{t}} \\ \Delta \mathrm{D}_{\mathrm{t}}\end{array}\right)$

If let

$\mathbf{B}=\left[\begin{array}{ccc}1+1 / \mathrm{N} & 0 & 0 \\ 0 & 1+1 / \mathrm{N} & 0 \\ 0 & 0 & 1 / \mathrm{F}-1\end{array}\right], \quad \mathbf{I}=\left[\begin{array}{ccc}1 & 0 & 0 \\ 0 & 1 & 0 \\ 0 & 0 & 1\end{array}\right]$

Formula (2.4) can be simplified as:

$\mathrm{P}_{\mathrm{c}}\left(\Delta \mathbf{E}_{\mathrm{t}}\right)=\mathbf{B} \mathbf{P}^{\mathrm{T}} \mathbf{I} \Delta \mathrm{E}_{\mathrm{t}}^{\mathrm{T}}$

\section{Emotion Generation Mechanism}

Different person will give different psychological response, which all attributes to various goals. If extern stimuli are in favor of pursuing the goal, one may response with greeting, admiration or joyness etc. Otherwise, one may response with hatred or distaste.

The explanation for emotion in psychology is that emotions are evaluations for oneself or for the relation status between agents and environment by human body [2]. We integrate the status of robot body and the extern environment. The status of robot body composes of emotion state, personality and basic survival condition. The basic survival condition denotes the amount of robot's energy. Fig 3.1 shows the frame of emotion generation mechanism.

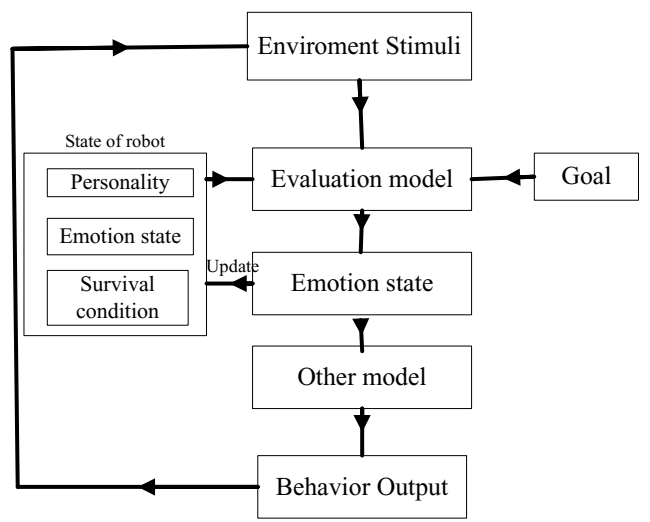

Fig 3.1 the frame of emotion generation system

In Fig 3.1, when robot perceives environmental stimuli, the environmental stimuli are sent to the evaluation model, in which the state of robot body and the goal are also considered at the same time. There is a priority problem between the need of current state of body and the current goal, that is to say, the agent should satisfy the survival condition first, and then finish the goal determined by human. After weighted by the evaluation model, the last emotion is updated by the current emotion. Finally, the output of robot changes the environment. Fig 3.2 illustrates the flow of the evaluation model. 


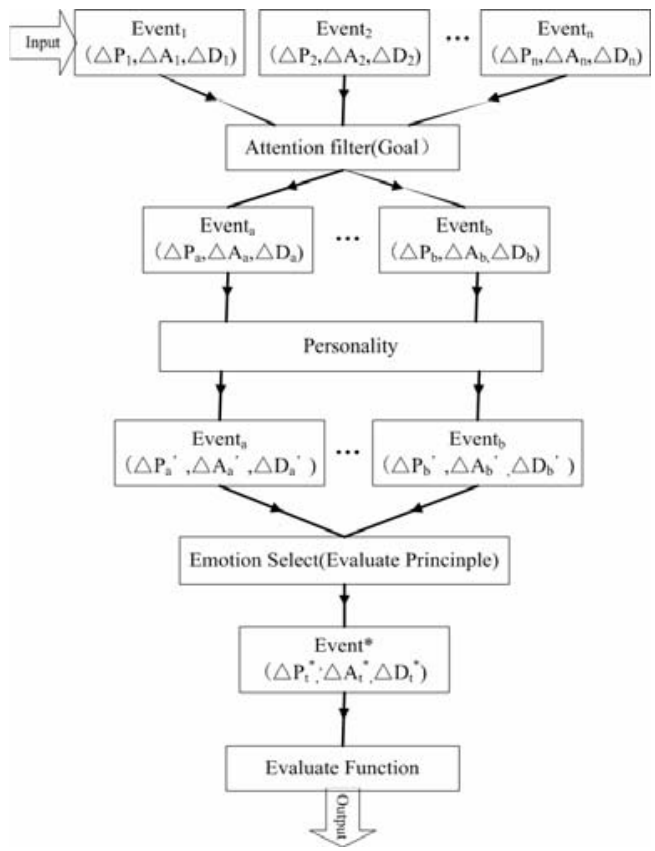

Fig 3.2 the flow of evaluation model

As in Fig 3.2, because of the inspiration from the OCC model, the extern stimuli are classified into several events according to the event features. At the beginning, the input events are Event ${ }_{1}$, Event $_{2}, \ldots$, Event $_{n}$, and the map from event to PAD emotion space is $\left(\Delta P_{i}, \Delta A_{i}, \Delta D_{i}\right)$, this vector is the objective change of emotion relative to event. The all events after filtered by the attention (decided by its goal), some events may be excluded, and the events reserved are Event ${ }_{1}, \ldots$, Event $_{n^{\prime}}$, where $n^{\prime} \leq n$; the objective emotion change caused by these reserved events converted into the robot's subjective emotion change; and then we choose an optimal event based on the value principle as the input of evaluation function. The method here used is described as follows:

$$
\begin{aligned}
& \text { Event } *=\text { Event }{ }_{i} \\
& \mathbf{E}_{t+1}=\mathrm{F}\left(\psi\left(\mathbf{E}_{t}\right)+\Delta \mathbf{E}_{t}\right) \\
& \Delta \mathbf{E}_{\mathrm{t}}=\left(\Delta \mathrm{P}_{\mathrm{t}}^{*}, \Delta \mathrm{A}_{\mathrm{t}}^{*}, \Delta \mathrm{D}_{\mathrm{t}}^{*}\right)
\end{aligned}
$$

Where $i=\arg \max _{k=1, \ldots, n^{\prime}}\left(\Delta P_{k}+\Delta A_{k}+\Delta D_{k}\right), \quad \Delta E_{t}=\left(\Delta P_{t}, \Delta A_{t}, \Delta D_{t}\right)$ is the delta of emotion at time t, $\psi$ is the function that represents how Emotion decays; $\mathrm{F}$ is the function that constrains the intensity of emotion between -1and 1 .

\section{Experiment Result and Analysis}




\subsection{Introduction of Fuwa Robot}

Fuwa robot is an amusement and service robot, which is developed by robotics institute of Civil Aviation University of China. Fuwa robot can be applied in the airport terminal, and the major task is comity and service. Not only has the autonomous navigation function of robot, but also has the following functions: welcoming guests with regards, dialoguing and handshaking with passenger, and performing the special show to the passenger etc.

\subsection{Experimental Design}

The available sensor information comprises the following three parts: (1) Face detection result through CCD camera image fuses with the result of the infrared sensor, and the finally fusing results judges whether the human exists. (2) The input of ultrasonic, which used to judge whether the obstacle exists in the process of navigation. (3) The input of person's voice signal through the microphone.

The change amount of robot emotion correlated to the various input. If the human do not interact with the robot in the long time, since the robot's drives of own task, the three dimensions of emotion will decrease. When the robot knows a person appearing in its vision, the robot emotion varies in each dimension along with the HRI degree, for instance, the emotion will change if the person says "You are so stupid", The detail of the change shows in Table 4.1.(the communication index denotes the degree of interaction).

Table 4.1.the map from the extern event to emotion change

\begin{tabular}{l|l|l|l}
\hline \multicolumn{1}{c|}{ Event } & $\triangle \mathrm{P}$ & $\triangle \mathrm{A}$ & $\triangle \mathrm{D}$ \\
\hline Person Coming & 0.05 & 0.05 & 0.05 \\
\hline Person going away & -0.1 & 0.2 & -0.15 \\
\hline Person saying"You are stupid" & -0.15 & 0.25 & -0.25 \\
\hline No person & -0.1 & -0.2 & -0.1 \\
\hline communication index 1 & 0.05 & 0.0 & 0.05 \\
\hline communication index 2 & 0.1 & 0.07 & 0.08 \\
\hline communication index 3 & 0.15 & 0.1 & 0.12 \\
\hline
\end{tabular}

Considering the robot should handshake with person, the map relationship from PAD emotion space to the action of handshake must be constructed. As the arm of robot with five degree of freedom (DOF), we allows for the two DOF of shoulder and the one DOF of elbow. Let the three angles of the three DOF are $\alpha, \beta, \gamma$ individually, and $t$ is the required time of completing the behaviour. The map function described as follows:

$\alpha=\alpha_{\text {Normal }}\left(0.5+\mathrm{e}_{\mathrm{d}}\right) ; \beta=\beta_{\text {Normal }}\left(0.5+\mathrm{e}_{\mathrm{d}}\right)$

$\gamma=\gamma_{\text {Normal }}\left(0.5+\mathrm{e}_{\mathrm{d}}\right) ; \mathrm{t}=\mathrm{t}_{\text {Normal }} /\left(0.5+\mathrm{e}_{\mathrm{v}}\right)$

$\mathrm{e}_{\mathrm{d}}=\frac{2 \mathrm{P}+\mathrm{D}}{6}+\frac{1}{2} ; \mathrm{e}_{\mathrm{v}}=\frac{\mathrm{A}}{2}+\frac{1}{2}$

Where $\alpha_{\text {Normal }}, \quad \beta_{\text {Normal }}, \gamma_{\text {Normal }}$ are the movement angles of normal state, and $t_{\text {Normal }}$ is the required time of finishing the behaviour in normal state. $\mathrm{e}_{\mathrm{d}}, \mathrm{e}_{\mathrm{v}} \in[0,1]$. 


\subsection{Experimental Process}

In order to affirm the validity of the proposed mechanism, the experiment is performed on the Fuwa robot. The Robot's initial emotion is set at the origin of the emotion space. Robot emotion varies according to the extent of communication. The robot interacts with a participant through speech and handshake. To manifest robot's different emotion, the participant requests to shake hands with the robot and the robot emotion can be showed from the responses.

Fig 4.1 shows the experimental process, when $t<60$, robot is in the idle state, and no person appears in its vision. When $t=60$, the participant comes to the front of the robot; When $60<\mathrm{t}<300$, due to the communication content is simple, thus, the communication index is high. When $t=240$, the participant requests to shake hands with robot for the first time. When $300<\mathrm{t}<360$, the communication index decreases because the communication content becomes complex. When $t=360$, the participant says "You are so stupid". When $t$ $=420$, the participant requests to shake hands with robot once more. When $t=480$, The participant goes away. When $480<\mathrm{t}<600$, No person communicates with the robot. When $\mathrm{t}=60$, we end the experiment.

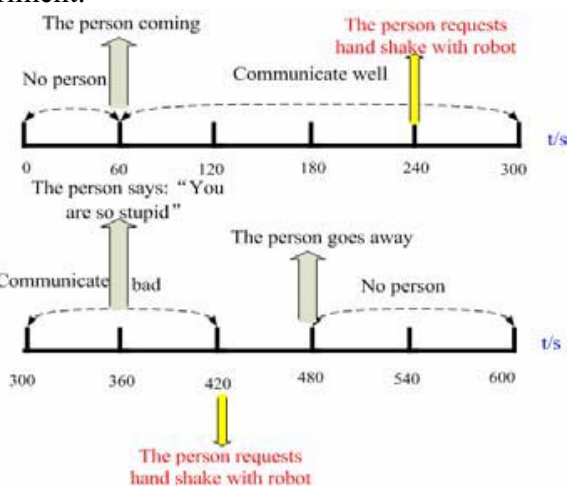

Fig 4.1 the experimental process

Two groups of personality parameter are set separately and are carried on the experiment twice according to the above experimental process.

\subsection{Result and Analysis}

Since the behaviour of handshake in the two experiments is similar, we only analyze the first experiment (Fig 4.2). The (a) of Fig 4.2 is the scene of the participant shaking hands with robot at the first time, and the (b) of Fig 4.2 is at the second time. Fig 4.3 and Fig 4.4 are emotion various curves with Personality = $(0.09,0.08,0.06)$ and Personality $=(0.2,-0.5,0.06)$ individually. 


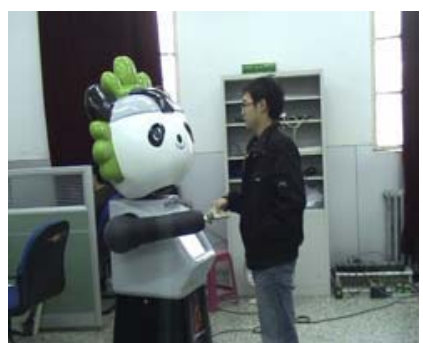

(a)

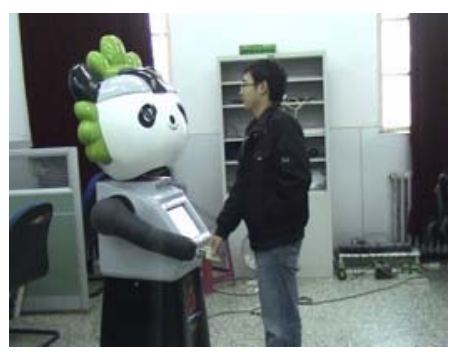

(b)

Fig 4.2 the robot shaking hands with human

As the two pictures of Fig 4.2 show, the angle of handshake in the Figure (a) is smaller than that in the Figure (b), that is because the interaction between robot and the participant is not fluent, therefore the robot displays unhappy emotion to shake hands with the participant.
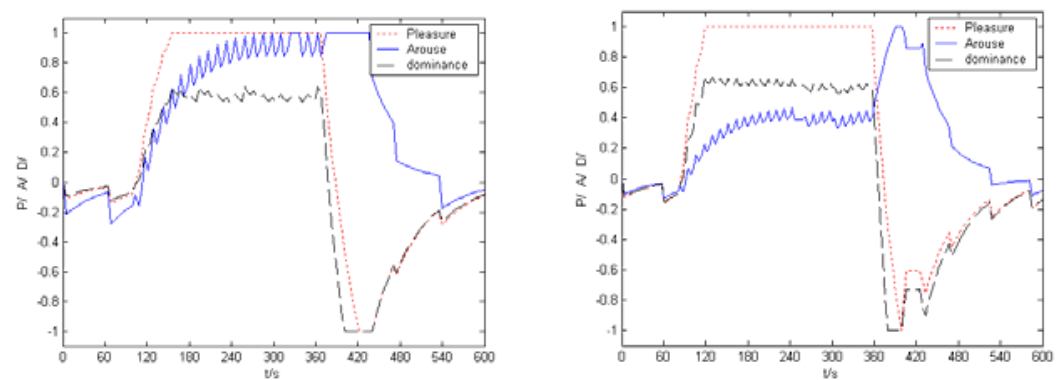

Fig 4.3 the various curve of emotion 1 Fig 4.4 the various curve of emotion 2

From the Fig 4.3 and Fig 4.4, we can see the tendencies of change are similar due to the same communication process. When $t<60$, since there is no person interacting with the robot, the three dimensions of emotion began to decrease from the origin. When $60<\mathrm{t}<$ 300 , although the robot sensed both the coming of person and the existing of obstacle, the robot decided to communicate with the person because the drive of its own task, and then the dimensions of robot emotion start to vary corresponding to the communication index. As the increase of the communication index, the three dimensions of emotion increased too. When $300<\mathrm{t}<420$, the robot emotion began to maintain invariable due to the decreasing of the communication index. When $t=360$, after the participant said" You are so stupid", the pleasure curve began to drop, and the dominance curve dropped since the robot was unable to control the situation, but the arouse curve ascend because of the intense stimulation. When $480<\mathrm{t}<600$, the three dimensions of emotion decayed to zero as the change of time because of the disappearing of the person. Even though the arouse curve had fluctuation, the entire curvilinear trend is reasonable, and may reflect the changing process of robot emotion. Because of the two groups of experiment with different personality, the emotion may be generated positive emotion easily. When $t=120$, the pleasure curve in Fig 4.4 has reached the maximum, while in the Fig 4.3 the pleasure curve need more time to reach the maximum. When the arouse curve in the Fig 4.4 reaches the peak value, the arouse curve reduces quickly, while in the Fig 4.4 maintains a period of 
time. The reason is that the neuroticism parameter of personality in the second time is less than the first time. The results suggest that we can change the parameter of personality to generate various emotions.

\section{Conclusions}

In this paper, a robot emotion generation mechanism is proposed, and emotion is described in PAD emotion space. In this mechanism, emotion is affected by the robot personality, the robot task and the emotion origin. The experiments demonstrate that the robot emotion can be changed naturally when it senses the extern stimuli. This mechanism can be applied in the robot with emotion system, so as to make the robot behaviour more flexible. The robot decision making involved with emotion generation mechanism is the later work.

\section{References}

[1]Broekens, J. and D. DeGroot. "Scalable and Flexible Appraisal Models for Virtual gents," CGAIDE, 2004.[11-++1]

[2] JIANG Dao-Ping, BAN Xiao-J uan, YIN Yi -Xin. Research on Emotion Theory and the Decision odels Based on Emotion .COMPUTER SCIENCE. 2007. 34(2)154157.[8+++2]

[3] Minsky M. The Society of Mind [M], New York, USA:Simon and Schuster, 1986.[2++3]

[4] Park, Cheonshu; Ryu, Jungwoo; Sohn, Joochan; Cho, Hyunkyu; An Emotion Expression System for the Emotional Robot, Consumer Electronics, 2007. ISCE 2007. IEEE International Symposium on Robot and Human Interactive Communication, 20-23 June 2007 Page(s): 1 - 6[3+++++4]

[5] Picard R W. Affective Computing [M]. MIT Press, London, England, 1997.[4++5]

[6]Rani, P, Sarkar, N.”Making Robots Emotion-Sensitive-Preliminary Experiments and Results," 14th IEEE International Workshop on Robot and Human Interactive Communication - ROMAN 2005[7---6]

[7] Sloman and Croucher, M. "Why Robot Will Have Emotions", In Proceedings IJCAI 1981, Vancouver.[1---7]

[8] SONG Yixu, J IA Peifa, A Control Architecture Based on Artificial Emotion for Anthropomorphic Robot, ROBOT.2004.29(4).491-495[5---8]

[9] WANG Guojiang, WANG Zhiliang, Survey of Artificial Emotion.Application Research Of Computers .2006(11).7-11[6+++9]

[10] WANG Guo-Jiang, WANG Zhi-Liang,CHEN Feng-Jun .Emotion Model of Interactive Virtual Humans Based on MDP. COMPUTER SCIENCE 2006, 12(33):135138.[12++10]

[11]Wei Zhehua. Research on Affective Computing of Emotional Robot Based Artificial sychology Theoy. [D].A Dissertation Submitted to Uninersity of Science and Technology Beijing For the Academic Degree of Master of Science, 2002[10++11]

[12]Youngmin Kim, Hyong-Euk Lee.Steward Robot: Emotional Agent for Subtle HumanRobot Interaction. The 15th IEEE International Symposium on Robot and Human Interactive Communication (RO-MAN06), Hatfield, UK, September 6-8, 2006[9++12] 